\title{
$\beta$-Amyloid Deposition Is Associated with Decreased Right Prefrontal Activation during Task Switching among Cognitively Normal Elderly
}

\author{
Hwamee Oh, Jason Steffener, Qolamreza R. Razlighi, Christian Habeck, Yaakov Stern \\ Cognitive Neuroscience Division, Department of Neurology, Columbia University College of Physicians and Surgeons, New York, New York 10032-3784
}

The accumulation of $\beta$-amyloid $(\mathrm{A} \beta)$ peptides, a pathological hallmark of Alzheimer's disease (AD), has been associated with functional alterations, often in an episodic memory system with a particular emphasis on medial temporal lobe function. The topography of $A \beta$ deposition, however, largely overlaps with frontoparietal control (FPC) regions implicated in cognitive control that has been shown to be impaired in early mild AD. To understand the neural mechanism underlying early changes in cognitive control with $\mathrm{AD}$, we examined the impact of $\mathrm{A} \beta$ deposition on task-evoked FPC activation using functional magnetic resonance imaging (fMRI) in humans. Forty-three young and 62 cognitively normal older adults underwent an fMRI session during an executive contextual task in which task difficulty varied: single (either letter case or vowel/consonant judgment task) vs dual (switching between letter case and vowel/consonant decisions) task. Older subjects additionally completed ${ }^{18} \mathrm{~F}$-florbetaben positron emission tomography scans and were classified as either amyloid positive $(\mathrm{A} \beta+)$ or negative $(\mathrm{A} \beta-)$. Consistent with previous reports, age-related increases in brain activity were found in FPC regions commonly identified across groups. For both task conditions, $A \beta$-related increases in brain activity were found compared with baseline activity. For higher cognitive control load, however, $\mathrm{A} \beta+$ elderly showed reduced task-switching activation in the right inferior frontal cortex. Our findings suggest that with $\mathrm{A} \beta$ deposition, brain activation in the cognitive control region reaches a maximum with lower control demand and decreases with higher control demand, which may underlie early impairment in cognitive control with AD progression.

Key words: amyloid PET; $\beta$-amyloid; cognitive control; cognitively normal older adults; fMRI; frontoparietal cortex

\section{Significance Statement}

The accumulation of $\beta$-amyloid $(\mathrm{A} \beta)$ peptides, a pathological hallmark of Alzheimer's disease, spatially overlaps with frontoparietal control (FPC) regions implicated in cognitive control, but the impact of A $\beta$ deposition on FPC regions is largely unknown. Using functional magnetic resonance imaging with a task-switching task, we found $\mathrm{A} \beta$-related increases in FPC regions compared with baseline activity. For higher cognitive control load, however, $\mathrm{A} \beta$-related hypoactivity was found in the right inferior frontal cortex, a region highly implicated in cognitive control. The findings suggest that with $\mathrm{A} \beta$ deposition, task-related brain activity may reach a plateau early and undergo downstream pathways of neural dysfunction, which may relate to the early impairment of cognitive control seen in the progression of $\mathrm{A} \beta$ pathology.

\section{Introduction}

Neuritic plaques of fibrillar $\beta$-amyloid (A $\beta$ ) peptides are hallmark characteristics of Alzheimer's disease (AD) pathology

Received Aug. 31, 2015; revised Dec. 7, 2015; accepted Dec. 31, 2015.

Author contributions: H.O., J.S., and Y.S. designed research; H.O., J.S., Q.R.R., and C.H. performed research; H.O., J.S., Q.R.R., and C.H. contributed unpublished reagents/analytic tools; H.O., Q.R.R., and C.H. analyzed data; H.O. and Y.S. wrote the paper.

This research was supported by the National Institute on Aging (Grant R01AG026158).

The authors declare no competing financial interests.

Correspondence should be addressed to Hwamee Oh, Cognitive Neuroscience Division, Department of Neurology and Taub Institute, Columbia University College of Physicians and Surgeons, 630 West 168th St., P\&S 16, New York, NY 10032. E-mail: hwamee.oh@columbia.edu.
(Braak and Braak, 1991; Hardy and Selkoe, 2002). Studies using positron emission tomography (PET) with radioligands binding to fibrillar $\mathrm{A} \beta$ plaques have found that $\sim 20-30 \%$ of cognitively normal older adults harbor $\mathrm{A} \beta$ deposition, consistent with autopsy findings (Bennett et al., 2006; Mintun et al., 2006). Even in cognitively normal elderly, the presence of elevated levels of $A \beta$ deposition has been associated with abnormal changes in multiple biomarkers, including brain gray matter atrophy, aberrant glucose metabolism, and reduced resting-state functional connectivity (Hedden et al., 2009; Becker et al., 2011; Mormino et al., 
2011; Johnson et al., 2014; Lowe et al., 2014; Oh et al., 2014). Task-related functional magnetic resonance imaging (fMRI) studies have commonly found $\mathrm{A} \beta$-related increases in brain activity during episodic memory (EM) performance (Sperling et al., 2009; Mormino et al., 2012; Oh and Jagust, 2013; Huijbers et al., 2014). In addition, this $\mathrm{A} \beta$-related increase in activity was shown to track memory strength in older adults with $\mathrm{A} \beta$ deposition (Elman et al., 2014). Together these results suggest that $\mathrm{A} \beta$ related changes in brain structure and function occur among cognitively normal elderly, which may herald behavioral trajectories of older adults in preclinical AD.

$\mathrm{A} \beta$-related functional changes have often been examined in a context of EM, with a particular focus on the medial temporal lobe (MTL) function. Topography of $\mathrm{A} \beta$ deposition, however, is quite widespread across the brain. Therefore, it is highly likely that $\mathrm{A} \beta$ deposition also affects other functional networks. A few recent studies have examined the impact of $A \beta$ deposition on neural systems beyond the EM network among cognitively normal elderly and early-stage probable AD subjects. Compared with normal controls, early-stage probable AD patients showed altered brain activation within the language system that was closely correlated with independently measured naming performance (Nelissen et al., 2007). In cognitively normal elderly, higher A $\beta$ deposition was related to hyperactivity in posterior left middle temporal gyrus, a region involved in associative-semantic language processing (Adamczuk et al., 2016). Previously, we examined the relationship between $\mathrm{A} \beta$ deposition and working memory load-related brain activation in cognitively normal elderly and found $\mathrm{A} \beta$-related increases in frontoparietal cortices with higher verbal working memory load (Oh et al., 2015). Therefore, emerging evidence seems to suggest that $A \beta$-related functional alterations are not specific to the EM network but occur in other functional networks as well.

The topography of $\mathrm{A} \beta$ deposition highly overlaps with regions known as frontoparietal control (FPC) regions that have been implicated in cognitive control, an ability to flexibly interact with the environment in accordance with an internal goal (Miller and Cohen, 2001; Braver et al., 2003; Brass and von Cramon, 2004; Badre and D'Esposito, 2007; Power et al., 2011; Cole et al., 2013; Waskom et al., 2014). Although A $\beta$-related and AD-related changes in cognition have often focused on EM, it is well documented that impairment of cognitive control is one of the early cognitive deficits observed in mild AD patients. Accumulating evidence further suggests that cognitive control impairment can be an early behavioral marker discriminating healthy aging from very mild $\mathrm{AD}$ dementia and predicting later conversion (Balota et al., 2010; Aschenbrenner et al., 2015). The neural mechanisms underlying cognitive control deficits in the early stage of $\mathrm{AD}$, however, remain largely unknown. One hypothesized mechanism is that $\mathrm{A} \beta$-related hyperactivation would reach a breakdown and fail to accommodate further cognitive processing demand, as postulated as neural reserve (Stern, 2009) and proposed by others (Jagust and Mormino, 2011). In the present study, we tested this hypothesis using an executive contextual task in which a level of cognitive control load varied. Specifically, we hypothesized that older adults with $\mathrm{A} \beta$ deposition $(\mathrm{A} \beta+)$, compared with older subjects without $A \beta$ deposition $(A \beta-)$, would show greater frontoparietal activation with lower control load, but decreased activity with higher control load. We further expected to observe age-related increases in frontoparietal activation as previously reported.

\section{Materials and Methods}

Participants. Forty-three healthy young adults (age range, 20-30 years; 29 females) and 62 cognitively normal older adults (age range, 60-70 years; 33 females) participated in the study. Subjects were recruited using a market mailing procedure. To ensure that subjects did not have dementia or mild cognitive impairment (MCI), a score of $\geq 136$ was required on the Mattis Dementia Rating Scale (DRS; Mattis, 1988). All subjects had no history of neurological and psychiatric illnesses and no major medical illness or medication that influenced cognition. Older subjects were classified as either amyloid positive $(A \beta+O)$ or amyloid negative $(A \beta-O)$ based on the criteria described below. All subjects provided informed consent in accordance with the Institutional Review Boards of the College of Physicians and Surgeons of Columbia University. Participants were paid for their participation in the study.

Neuropsychological tests. A comprehensive battery of neuropsychological (NP) tests were administered to all participants. Using a subset of NP tests, cognitive composite scores for processing speed/attention and EM were generated: for processing speed/attention (NP-process), the Wechsler Adult Intelligence Scale-Third Edition Digit Symbol subtest (Wechsler, 1997), the Trail Making Test Part A (Reitan, 1978; inversed value), and the Stroop Color Naming Test-Color Naming in $90 \mathrm{~s}$ (Golden, 1978) were included; for EM (NP-memory), scores from the Selective Reminding Test (SRT; Buschke and Fuld, 1974)—Long-Term Storage, SRT - Continued Recall, and SRT—Recall at the Last Trial were combined. The American National Adult Reading Test (AMNART; Grober and Sliwinski, 1991) was used as an estimate of IQ.

Experimental design. The executive contextual task used for fMRI was adapted from Koechlin et al. (2003). A schematic diagram of the task design is provided in Figure $1 \mathrm{~A}$. Subjects were presented a series of task blocks that comprised two single-task conditions and two identical dualtask conditions, each implemented in blocks. Each block lasted $33.5 \mathrm{~s}$ in which 12 letters were displayed sequentially. At the beginning of each block, an instruction page was shown for $4.7 \mathrm{~s}$ to inform subjects of the appropriate action for each stimulus. The single-task condition was either a vowel/consonant judgment task or a lower-case or upper-case judgment task that was determined based on colors of letters. In the vowel/consonant judgment task, either a green or a white letter appeared; a green letter was associated with the vowel/consonant task (left press for vowel, right press for consonant), while white letters required no action. In the lower/upper-case judgment task, a red letter signaled lower/uppercase judgment (left press for lower case; right press for upper case); with white letters, no action was required. In the dual-task condition, both green and red letters appeared together with white letters. White letters comprised one third of letter stimuli in each block (i.e., four letters). Each stimulus was shown for $\leq 2400 \mathrm{~ms}$, but terminated following a response before that deadline. A blank screen was inserted between trials so that the stimulus-onset asynchrony was fixed as $2.4 \mathrm{~s}$. In addition to the active task blocks, there was a resting-condition block, $33.5 \mathrm{~s}$ in duration, during which no stimuli was presented and no response was required. Each functional run consisted of four task (two single-task blocks and two dual-task blocks) and two resting-condition blocks; in total, the scan session was composed of six functional runs. Before scanning, subjects were given 36 block sessions for practice to stabilize their performance. The executive contextual task fMRI session lasted $\sim 26 \mathrm{~min}$.

Response time (RT) of correct trials and proportion correct for singletask and dual-task conditions were calculated for each individual and reported as behavioral measures.

MRI data acquisition. Participants underwent MRI using a 3T Philips Achieva System equipped with a standard quadrature headcoil. Highresolution T1-weighted magnetization-prepared rapid gradient echo scans were collected axially for each subject $[\mathrm{TR}=6.6 \mathrm{~ms}$; TE $=3 \mathrm{~ms}$; flip angle, $8^{\circ}$; field of view (FOV), $256 \times 256 \mathrm{~mm}$; matrix size, $256 \times 256 \mathrm{~mm}$; slices, 165; voxel size, $1 \times 1 \times 1 \mathrm{~mm}^{3}$ ]. For the executive context task scans, 111 volumes of functional images were acquired in each run (six runs in total) using a $\mathrm{T} 2{ }^{\star}$-weighted gradient-echo echo planar image sequences $\left(\mathrm{TR}=2000 \mathrm{~ms}\right.$; $\mathrm{TE}=20 \mathrm{~ms}$; flip angle, $72^{\circ}$; FOV, $224 \times 224$ $\mathrm{mm}$; voxel size, $2 \times 2 \mathrm{~mm}$; slice thickness, $3 \mathrm{~mm}$; duration, $3.5 \mathrm{~min}$ ). Each functional volume consisted of 41 transverse slices per volume. 
Four dummy volumes acquired at the beginning of each functional run were discarded from the dataset before image processing and analysis.

Structural MRI image processing. For each subject, a single structural T1 image was processed through FreeSurfer v5.1 to implement region-of-interest (ROI) labeling following the FreeSurfer processing pipeline (http://surfer. nmr.mgh.harvard.edu/). Briefly, structural images were bias field corrected, intensity normalized, and skull stripped using a watershed algorithm, followed by a white matter-based segmentation, defining gray/white matter and pial surfaces, and topology correction (Dale et al., 1999). Subcortical and cortical ROIs spanning the entire brain were defined in each subject's native space (Fischl et al., 2002).

${ }^{18} \mathrm{~F}$-florbetaben-PET acquisition, image processing, and analysis. ${ }^{18} \mathrm{~F}$-florbetaben was donated by Piramal (Piramal Pharma). PET scans were performed using a Siemens MCT PET/CT scanner in dynamic, three-dimensional acquisition mode. Dynamic acquisition frames were obtained over $20 \mathrm{~min}(4 \times 5 \mathrm{~min}$ frames $)$ beginning $50 \mathrm{~min}$ following the bolus injection of $10 \mathrm{mCi}$ of ${ }^{18} \mathrm{~F}$-florbetaben. An accompanying structural CT scan (in-plane resolution, $0.58 \times$ $0.58 \mathrm{~mm}$; slice thickness, $3 \mathrm{~mm}$; FOV, $300 \times$ $300 \mathrm{~mm}$; number of slices, 75) was acquired and used for attenuation correction. PET data were reconstructed using a TrueX (HD-PET) algorithm. Images were smoothed with a $2 \mathrm{~mm}$ Gaussian kernel with scatter correction.

Dynamic PET frames (four scans) were aligned to the first frame using rigid-body registration and a static PET image was obtained by averaging the four registered frames. The static PET and CT images were coregistered and merged to generate a composite image in the PET static space. Each individual's structural T1 image in FreeSurfer space was also registered to the participant's merged image to transfer ROIs (see below) and the cerebellar gray matter from FreeSurfer space to static PET image space. These ROIs in static PET space were used to extract the regional PET data.

The standardized uptake value (SUV) was calculated at selected cortical regions that have been also adopted for the processing stream of the Alzheimer's Disease Neuroimaging Initiative (adni.loni.usc.edu; Landau et al., 2013,2015). The SUV was then normalized to gray matter cerebellum to derive the SUV ratio (SUVR). The selected regions included the following: the frontal (all frontal regions anterior to the precentral sulcus), the temporal (superior and middle temporal gyri), the parietal (superior and inferior parietal cortices, supramarginal gyrus, and precuneus), and the anterior/posterior cingulate cortices. Mean SUVR values from these large ROIs constituted a global amyloid index for each subject.

Amyloid positivity of elderly subjects was determined using a k-means clustering method as previously reported (Oh et al., 2015). Twelve older subjects were classified as $\mathrm{A} \beta+; 50$ older subjects were classified as $\mathrm{A} \beta-$. The proportion of $\mathrm{A} \beta+$ subjects in our sample is comparable to the reports from other studies (Mormino, 2014).

fMRI image processing and analysis. All fMRI analyses were performed with SPM8 (Wellcome Department of Imaging Neuroscience, London, UK). Preprocessing steps included slice timing, motion correction, coregistration of the T1-weighted anatomical image and the mean functional image, spatial normalization to the Montreal Neurological Institute template, and smoothing with an isotropic Gaussian kernel of $8 \mathrm{~mm}$ full-width half-maximum.

For the whole-brain analysis of the executive contextual task, a blockbased analysis was performed for each participant. A subject-level design matrix was constructed using a GLM consisting of epochs representing each experimental task block: two single-task blocks, two dual-task blocks, and two rest blocks in each run, six functional runs in total. Task blocks in each run were convolved with the canonical hemodynamic response function in SPM8 (i.e., a double-gamma function) for the block duration. In addition to the task block regressors, time-series BOLD signals were high-pass filtered $(1 / 128 \mathrm{~Hz})$ to remove low-frequency drifts. Because the fMRI data were analyzed as a block design, we did not separately model motion-related parameters (i.e., six rigid-body translation and rotation parameters) that did not show any group differences across three subject groups (Johnstone et al., 2006). To identify brain regions that show task-related activity common across groups, three group maps were created by comparing single task versus baseline and dual task versus baseline, and contrasting single task versus dual task collapsing across groups at the group-level analysis, by applying a onesample $t$ test, controlling for the age group. The whole-brain family-wise error was cluster-corrected to $p<0.05$ (two-sided) using a clusterforming threshold of $p<0.05$. Thresholded statistical maps were projected onto inflated atlases for display purposes using Caret v5.65 software. To assess the effect of age and $\mathrm{A} \beta$ deposition on task-related activity in single-task and dual-task conditions and activity difference between dual-task versus single-task conditions, ROI analyses were conducted by extracting mean contrast values from all suprathreshold voxels of task-positive and task-negative brain regions from each group map using the MarsBar Matlab toolbox (Brett et al., 2002; http://marsbar. sourceforge.net). Extracted mean contrast values were averaged for taskpositive and task-negative regions separately. These values were then used in ANOVA to assess group differences using SPSS v.22. Age and sex were controlled in all analyses when only older subjects were included. 
Table 1. Subject characteristics

\begin{tabular}{|c|c|c|c|c|c|c|c|c|}
\hline & \multicolumn{2}{|l|}{ Young } & \multicolumn{2}{|l|}{ All old } & \multicolumn{2}{|l|}{$A \beta-0$} & \multicolumn{2}{|l|}{$A \beta+0$} \\
\hline & Mean & SD & Mean & SD & Mean & SD & Mean & SD \\
\hline N & 43 & & 62 & & 50 & & 12 & \\
\hline Age (years) & 26.6 & 4.9 & 64.6 & 3 & 64.7 & 2.9 & 64.2 & 3.5 \\
\hline Female [N (\%)] & $29(67 \%)$ & & $33(53 \%)$ & & $27(54 \%)$ & & $6(50 \%)$ & \\
\hline Education $^{a}$ (years) & 15.6 & 1.9 & 16.7 & 2.4 & 16.9 & 2.4 & 16.8 & 2.3 \\
\hline Global SUVR & & & & & 1.15 & 0.05 & 1.37 & 0.12 \\
\hline Mattis DRS score & 140 & 2.3 & 139.8 & 2.5 & 139.4 & 2.8 & 140.4 & 2.3 \\
\hline AMNART score & 29.9 & 7.7 & 33.7 & 9.7 & 33.5 & 9.7 & 35.5 & 8.2 \\
\hline $\begin{array}{l}\text { NP-process composite } \\
\text { score }\end{array}$ & 0.5 & 1.3 & -0.1 & 3.2 & -0.1 & 3.5 & -0.2 & 0.9 \\
\hline $\begin{array}{l}\text { NP-memory composite } \\
\text { score }^{b}\end{array}$ & 0.9 & 2.4 & -0.4 & 1.7 & -0.5 & 1.9 & -0.4 & 0.6 \\
\hline
\end{tabular}

${ }^{a}$ Older subjects were more educated than young subjects $(p<0.01)$.

${ }^{b}$ Young subjects performed better than older subjects $(p<0.005)$.

To assess $A \beta$-related differences in task-related activity with a finer grain, we additionally performed direct voxelwise group comparisons between $\mathrm{A} \beta+$ and $\mathrm{A} \beta-$ groups in three activation maps: single-task versus baseline, dual-task versus baseline, and single-task versus dualtask contrast maps. These whole-brain voxelwise comparisons would complement ROI analyses that are more robust to multiple-comparison corrections, while detecting the brain regions most robustly representing the association with $\mathrm{A} \beta$ deposition.

\section{Results}

\section{Subject characteristics}

Subject data are summarized in Table 1. Older subjects were more educated than young subjects $\left(T_{(103)}=-2.8, p<0.01\right)$. $\mathrm{A} \beta+\mathrm{O}$ vs $\mathrm{A} \beta-\mathrm{O}$ groups did not differ in age, gender, education, DRS scores, or AMNART scores. With composite NP test scores, young subjects, compared with $\mathrm{A} \beta-\mathrm{O}$ subjects, showed significantly higher NP-memory scores, while no other group differences were found for both NP-memory and NP-process composite scores.

\section{Behavioral results of executive contextual task}

With RT, a $2 \times 3$ ANOVA with task condition (single-task vs dual-task conditions) and group (young, $\mathrm{A} \beta-\mathrm{O}$, and $\mathrm{A} \beta+\mathrm{O}$ ) being independent variables revealed significant main effects of task condition and group: for task condition, $F_{(1,200)}=106.96$, $p<0.001$; for group, $F_{(2,200)}=38.85, p<0.001$. Post hoc analyses revealed that young subjects were significantly faster than older subject groups across all conditions ( $p$ 's $<0.05$ ). An interaction effect between task condition and group approached significance $\left(F_{(2,200)}=2.87, p=0.06\right)$. With accuracy rate, assessed by a $2 \times$ 3 ANOVA, neither main nor interaction effect was significant ( $p$ 's $>0.1$ ), indicating that accuracy measures (i.e., proportion correct) were not different across groups ( $p$ 's $>0.1)$. $\mathrm{A} \beta+\mathrm{O}$ and $\mathrm{A} \beta-\mathrm{O}$ groups did not differ in any behavioral measures of the fMRI task. The results are shown in Figure $1 B$.

\section{Age-related increases in single-task and dual-task activations compared with baseline}

To assess a differential effect of age and $\mathrm{A} \beta$ deposition on brain activity in frontoparietal regions implicated in cognitive control, we first examined the effect of age on brain activity during the executive contextual task by comparing healthy young and $\mathrm{A} \beta-\mathrm{O}$ subjects. Age-related brain activity patterns were examined in single-task and dual-task conditions compared with baseline and a contrast of dual-task versus single-task conditions (i.e., parametric increases or decreases due to executive control load) separately. Task-related activation (task-positive) was found
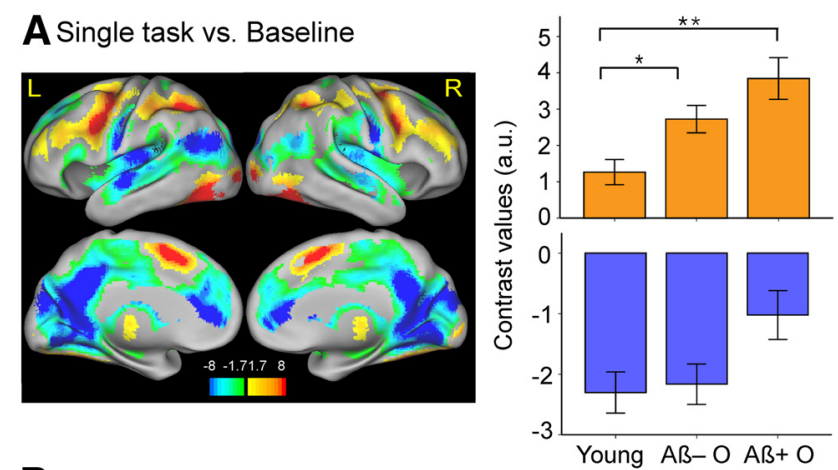

\section{B Dual task vs. Baseline}

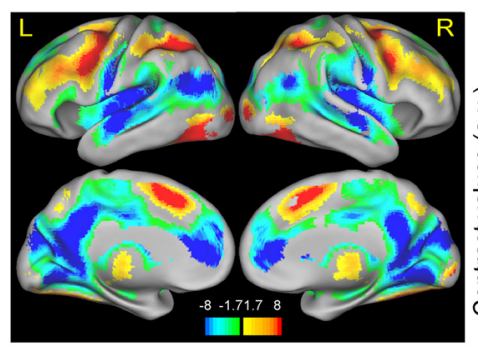

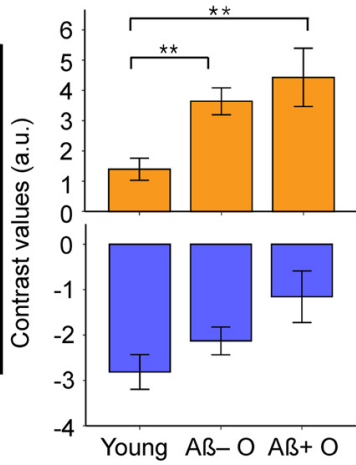

Figure 2. Single-task and task-switching fMRI results by group. $A$, Brain activation during the single-task condition compared with baseline shows an age-related increase in taskpositive regions, independent of $A \beta$ deposition. Plots display mean contrast values of significant clusters showing task-related activation (i.e., warm-colored regions in the left panel) and deactivation (i.e., cool-colored regions in the left panel) for each group. $\boldsymbol{B}$, Brain activation during the dual task compared with baseline shows an age-related increase in task-positive regions, independent of $A \beta$ deposition. Plots display mean contrast values of significant clusters showing task-related activation (i.e., warm-colored regions in the left panel) and deactivation (i.e., cool-colored regions in the left panel) for each group. Brain activation maps are thresholded at $p<0.05$, cluster corrected for multiple comparisons. Scales represent $T$ values. $\mathrm{L}$, Left hemisphere; $\mathrm{R}$, right hemisphere. Error bars in the bar charts represent SEM. ${ }^{*} p<0.05$; ${ }^{* *} p<0.01$.

throughout the lateral frontal and parietal cortices, the superior medial frontal cortex, and the lateral temporo-occipital cortices bilaterally in the single-task condition compared with baseline (Fig. 2). Task-related deactivation (task-negative) was found in the lateral temporal and temporoparietal cortices bilaterally, and in the medial frontal and parietal cortices. Compared with young subjects, older subjects, independent of $A \beta$ status, showed greater activity in task-positive regions, while no $\mathrm{A} \beta$-related difference was found in task-positive regions ( $p>0.05$; Fig. $2 A$, bar charts). In task-negative regions, no group difference was found. Similar topography of task-related activation and deactivation was found in the dual-task condition compared with baseline activity (Fig. $2 B)$. In addition, a significant age effect was found such that independent of $\mathrm{A} \beta$ deposition, older subjects showed increased task-positive activation compared with young subjects, while no group difference was found in task-negative activation. None of these activations was correlated with RT or accuracy measures collapsing all subject groups.

\section{A $\beta$-related increases in single-task and dual-task activations compared with baseline}

To further assess $\mathrm{A} \beta$-related differences in brain activity supporting executive control with higher resolution that might have been missed in our ROI analyses, we directly compared $\mathrm{A} \beta+\mathrm{O}$ and $\mathrm{A} \beta-\mathrm{O}$ groups in brain activation obtained from three contrast 
$A A \beta+O>A \beta-O$ in Single task vs. Baseline
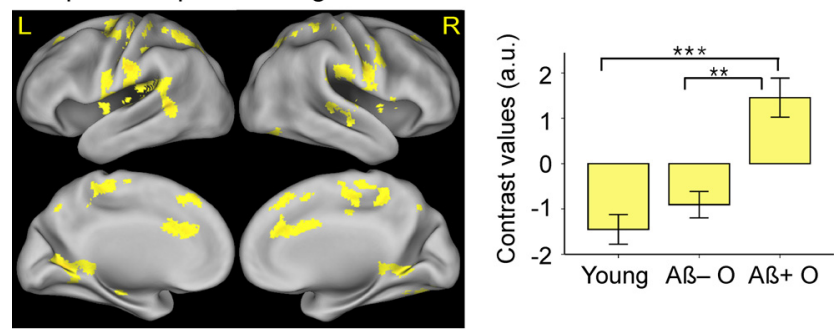

B $A \beta+O>A \beta-O$ in Dual task vs. Baseline
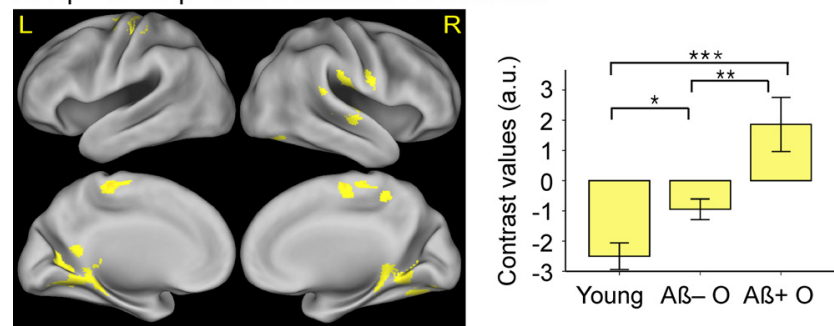

Figure 3. $A \beta$ deposition is associated with increased brain activity in subregions that deactivate commonly across all subjects during the executive contextual task conditions. $A, A \beta$ related increases in brain activity for the single task compared with baseline. $B, A \beta$-related increases in brain activity for the dual task compared with baseline. These regions mostly overlap with task-related deactivation regions common across all subjects as shown in Figure $2 A, B$. The whole-brain voxelwise contrast maps between $A \beta+$ and $A \beta-$ groups are thresholded at $p<0.05$, cluster corrected for multiple comparisons. Scales represent $T$ values. L, Left hemisphere; $R$, right hemisphere. Plots display mean contrast values of significant clusters showing $A \beta$-related increases in brain activation for each group. Contrast values of young subjects from the suprathreshold clusters are displayed for comparison purposes. Error bars in the bar charts represent SEM. ${ }^{*} p<0.05 ;{ }^{* *} p<0.01 ;{ }^{* * *} p<0.001$.

maps: single task versus baseline; dual task versus baseline; and a contrast of dual-task versus single-task conditions. These wholebrain voxelwise analyses revealed that in single-task compared with baseline activity, $A \beta$-related increases in activity were found in the anterior cingulate cortex, the posterior hippocampus, the superior medial frontal cortex, the lateral temporal cortex, the superior parietal cortex, the primary sensory and motor cortex, and the insular cortex (Fig. $3 A$ ). A $\beta$-related increases in activity in dual-task compared with baseline activity were found, although less extensive, in the right temporal cortex, the posterior hippocampus, and the primary sensorimotor cortex (Fig. $3 B$ ). When we restricted these $\mathrm{A} \beta$-related activation patterns within the common control regions identified across all subject groups, $\mathrm{A} \beta$ related increases were more prominent in task-negative regions (Fig. $3 A, B$ ). No significant voxel was found in the opposite contrasts (i.e., greater activity for $\mathrm{A} \beta-\mathrm{O}$ than $\mathrm{A} \beta+\mathrm{O}$ ). As expected, young subjects showed task-negative activation within regions showing $A \beta$-related increases in activity, which was significantly greater than that in $\mathrm{A} \beta+$ older subjects for the single-task condition and those in both $\mathrm{A} \beta+\mathrm{O}$ and $\mathrm{A} \beta-\mathrm{O}$ groups for the dualtask condition (Fig. $3 A, B$, bar charts). The categorical differences in brain activity between $\mathrm{A} \beta+\mathrm{O}$ and $\mathrm{A} \beta-\mathrm{O}$ groups were further confirmed by significant associations between brain activity and global amyloid indices treated as a continuous variable, controlling for age and sex (single-task vs baseline activity: $\beta=0.26, p<$ 0.05 ; dual-task vs baseline activity: $\beta=0.26, p<0.05)$. When frontal and parietal cortical SUVRs were associated with brain activity in these regions, the results remained similar to those obtained from global amyloid indices with a stronger trend with parietal than frontal cortical SUVRs (for single task vs baseline: frontal SUVR: $\beta=0.23, p=0.07$; parietal SUVR: $\beta=0.29, p<$
0.05; for dual task vs baseline: frontal SUVR: $\beta=0.24, p=0.06$; parietal SUVR: $\beta=0.31, p<0.05$ ).

Age-related increases but $\mathrm{A} \beta$-related decreases in brain activity in task-positive regions with increased demand of cognitive control

To assess parametric changes in brain activity related to cognitive control load (i.e., dual-task vs single-task conditions), we first identified brain regions that showed parametric increases and decreases commonly across young, $\mathrm{A} \beta-\mathrm{O}$, and $\mathrm{A} \beta+\mathrm{O}$ groups, controlling for age group and performance (Fig. 4A). Loadrelated parametric increases in activity common across groups were found in lateral frontal and lateral parietal cortices bilaterally, the medial parietal cortex, the inferior temporal cortex, and the precuneus. Parametric decreases related to cognitive control load were found in the medial frontal cortex, the lateral temporoparietal cortices, the insular cortex, and the left hippocampus. To assess age-related and $\mathrm{A} \beta$-related changes in parametric modulation in activity due to cognitive control load, we extracted contrast values from the suprathreshold clusters identified as cognitive control-related parametric increases and decreases in activity and compared them between groups. Compared with young subjects, $\mathrm{A} \beta-\mathrm{O}$ subjects showed greater load-related parametric increases in the identified brain regions (Fig. $4 B$, upper bar chart). The $\mathrm{A} \beta+\mathrm{O}$ group, however, did not differ from the $\mathrm{A} \beta-\mathrm{O}$ group in parametric increases in activity collapsing across the identified task-positive regions, controlling for age and sex. In brain regions showing parametric decreases in activity in association with cognitive control load, no group difference was observed (Fig. 4B, lower bar chart).

To further assess which task-related activity derived the observed group differences in parametric activity slope, we masked the brain activation maps for the single-task vs baseline activity and dual-task vs baseline activity with the task-positive and tasknegative network maps derived from the dual-task vs single-task contrast common across all three groups (i.e., Fig. 4A). Extracted mean activity values for the single-task and dual-task conditions compared with baseline activity from these masked regions are plotted by subject groups (Fig. 4C). The line charts in Figure $4 C$ demonstrate that task-positive activity for both single-task and dual-task conditions compared with baseline increase with age and $\mathrm{A} \beta$ deposition, while the activity difference between dualtask and single-task conditions was the greatest in the $\mathrm{A} \beta-\mathrm{O}$ group.

To further determine age-related and $\mathrm{A} \beta$-related activity differences in cognitive control with higher resolution, we conducted a whole-brain voxelwise analysis by directly comparing $\mathrm{A} \beta-\mathrm{O}$ and $\mathrm{A} \beta+\mathrm{O}$ groups in control load-related brain activity. This analysis revealed that, compared with the $\mathrm{A} \beta-\mathrm{O}$ group, the $\mathrm{A} \beta+\mathrm{O}$ group showed parametric decreases in activity for cognitive control in the right prefrontal cortex and the right insular cortex (Fig. 5). Plots displaying mean contrast values of significant clusters are provided to better visualize load-related parametric decreases in activity in $\mathrm{A} \beta+\mathrm{O}$ compared with $\mathrm{A} \beta-\mathrm{O}$ subjects (Fig. 5). By treating global amyloid indices as a continuous variable, we found a similar association between $\mathrm{A} \beta$ deposition and right prefrontal activation $(\beta=-0.34, p=0.005)$. The results remained the same when we assessed the relationships using frontal and parietal cortical SUVRs (frontal SUVR: $\beta=$ $-0.32, p<0.01$; parietal SUVR: $\beta=-0.31, p<0.05$ ). Brain regions showing $\mathrm{A} \beta$-related decreases in cognitive control activity also showed an age-related difference such that, compared 


\section{A Dual vs. Single task}

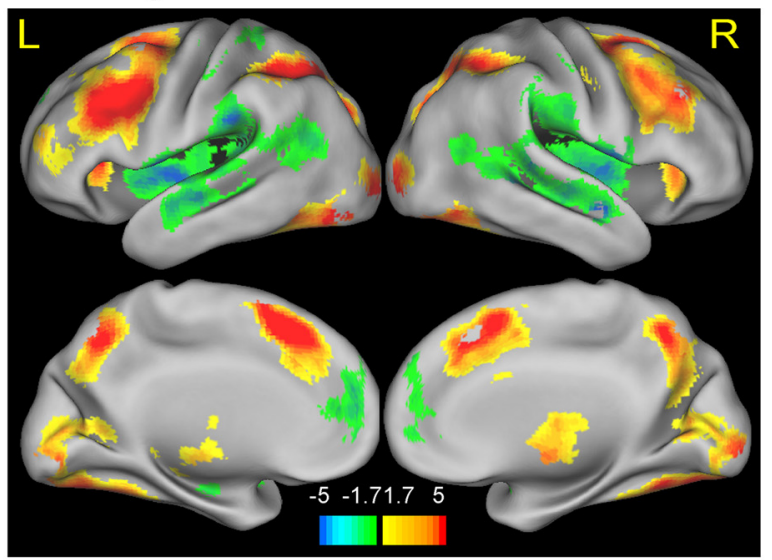

B
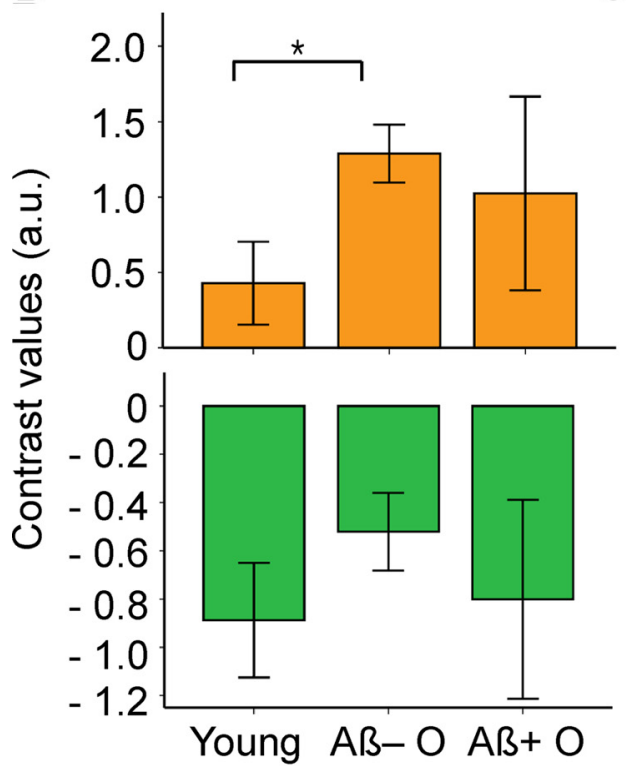

C
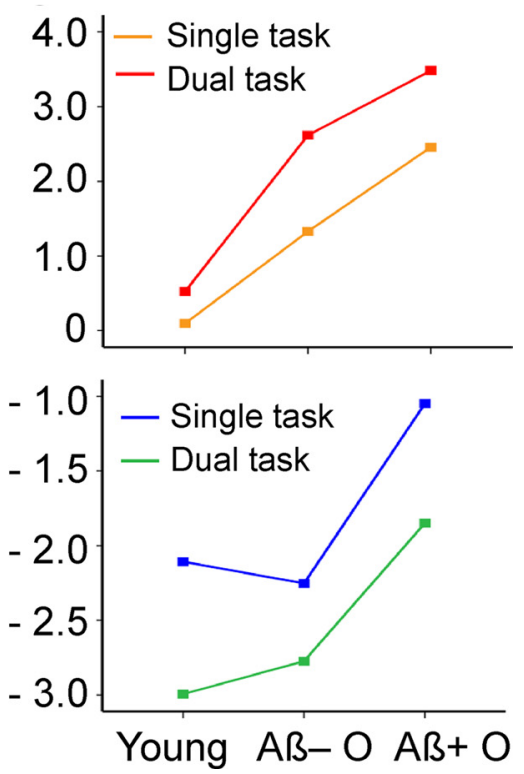

Figure 4. Age is associated with increased brain activity with higher cognitive control load compared with lower cognitive control load conditions. $\boldsymbol{A}$, Brain regions demonstrating activation and deactivation in relation to higher cognitive control load commonly across all subject groups. Brain activation maps are thresholded at $p<0.05$, cluster corrected for multiple comparisons. Scales represent $T$ values. L, Left hemisphere; $\mathrm{R}$, right hemisphere. $\boldsymbol{B}$, Age is associated with greater increases with a higher cognitive control load in task-positive activation in the cognitive control regions common across all subjects. Plots display mean contrast values of significant clusters showing parametric increases with cognitive control load (i.e., yellow/red-colored regions in $\boldsymbol{A}$; task-positive regions) and parametric decreases with cognitive control load (i.e., green/blue-colored regions in $\boldsymbol{A}$; task-negative regions) for each group. Error bars represent SEM. ${ }^{*} p<0.05$. C, Line charts represent mean contrast weights compared with baseline within cognitive control load-related task-positive and task-negative regions by cognitive control load condition and group. Red line: Dual-task, task-positive regions; orange line: single-task, task-positive regions; green line: dual-task, tasknegative regions; blue line: single-task, task-negative regions.

with young subjects, $\mathrm{A} \beta-\mathrm{O}$ subjects showed increased activity for cognitive control.

To assess which task activity derived the observed group differences in parametric activity slope within these regions, we extracted mean contrast values from the suprathreshold clusters for the single-task versus baseline activity and dual-task vs baseline activity separately and plotted them by group. Line charts in Figure $5 C$ demonstrate that for the single task, activity continues to increase with age and $\mathrm{A} \beta$ deposition, while for the dual task, activity increases with age, followed by decreases with $\mathrm{A} \beta$ deposition. Within the $A \beta+$ group, right inferior frontal cortex activation was further significantly correlated with RT difference between dual-task and single-task conditions, indicating that longer RT for the dual-task compared with single-task conditions relates to greater activation in right inferior frontal cortex $(\beta=0.8, p<0.01)$.

\section{Discussion}

In this study, we examined the association between fibrillar $\mathrm{A} \beta$ deposition and taskrelated frontoparietal activation during cognitive control among cognitively normal elderly. Several key findings emerged: (1) we replicated age-related frontoparietal hyperactivation during cognitive control, independent of $\mathrm{A} \beta$ deposition; (2) while providing additional support for $\mathrm{A} \beta$-related hyperactivation in cognitively normal elderly, we showed that $\mathrm{A} \beta$-related hyperactivation is not restricted to the EM system but also occurs in the cognitive control brain regions; (3) $\mathrm{A} \beta$ related activity in the right prefrontal cortex, which was thought to be critical in cognitive control, decreased with higher cognitive control load, while overall being greater compared with baseline activity.

Approximately 20 30\% of cognitively normal elders harbor elevated accumulation of $\mathrm{A} \beta$, which has been considered as a cause of downstream pathological events during the progression of $\mathrm{AD}$ (Selkoe, 2003). Because of the relevance of $A \beta$ deposition to $\mathrm{AD}$ and the most detrimentally affected cognitive function in $\mathrm{AD}$ patients being EM, studies investigating the relationship of $\mathrm{A} \beta$ deposition with other neural markers have focused primarily on the brain systems supporting EM, with a particular focus on MTL, including the hippocampus and more directly anatomically connected regions. The topographical distribution of $\mathrm{A} \beta$ deposition, however, is quite widespread across the brain and overlaps highly with brain regions supporting cognitive control, such as the prefrontal and parietal cortices (Braak and Braak, 1991). Therefore, it is highly likely that $\mathrm{A} \beta$ deposition also affects other cognitive and memoryrelated networks.

It is well established that the prefrontal cortex is involved in the executive/cognitive control process. Cognitive control refers to an ability to flexibly interact with the environment by adopting task-relevant behavior while ignoring task-irrelevant one and has been considered to underlie several higher cognitive processes. Lesion, electrophysiological, and fMRI studies in humans and nonhuman primates have shown that the prefrontal cortex, and the lateral frontal cortex in particular, is a critical neural substrate subserving this process. With the damage or reduced brain activation in the prefrontal cortex, subjects' performance in cognitive control function was undermined. The lateral parietal cortex is also thought to be commonly involved in control by showing taskrelated increases in brain activity as measured in fMRI (Braver et 
A

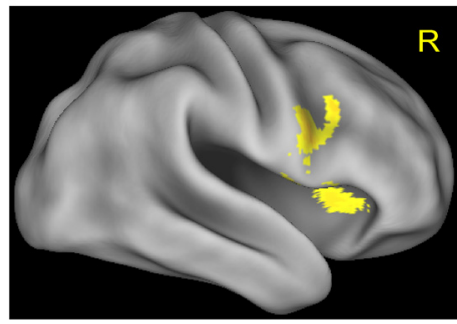

B

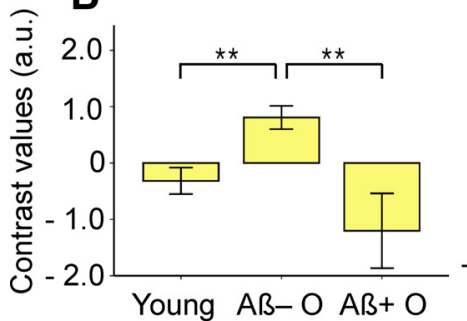

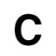

2.5 - Single task

2.0 - Dual task

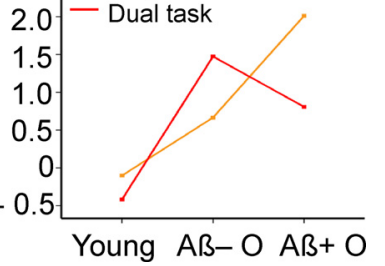

Figure 5. $A \beta$ deposition is associated with decreased brain activity in the right prefrontal and insular cortices with higher cognitive control load compared with lower cognitive control load. $A, A \beta$-related decreases in brain activity for the dual-task compared with single-task conditions. These regions mostly overlap with task-related activation regions common across all subjects as shown in Figure $4 A$. The whole-brain voxelwise contrast maps between $A \beta+$ and $A \beta-$ groups are thresholded at $p<0.05$, cluster corrected for multiple comparisons. $\boldsymbol{B}$, Plots display mean contrast values of significant clusters showing $A \beta$-related decreases in brain activation for each group. Contrast values of young subjects from the suprathreshold clusters are displayed for comparison purposes. Error bars in the bar charts represent SEM. ${ }^{* *} p<0.01$. $C$, Line charts represent mean contrast weights compared with baseline within regions showing $\mathrm{A} \beta$-related decreases in brain activation by cognitive control load condition and group. Red line: Dual-task, task-positive regions; orange line: single-task, task-positive regions.

al., 2003; Brass and von Cramon, 2004; Badre and D'Esposito, 2007). Studies of resting-state and task-based connectivity analyses (Power et al., 2011; Cole et al., 2013) further suggest that control is supported in part by a distributed frontoparietal network centered on the inferior frontal sulcus and intraparietal sulcus (Waskom et al., 2014), highlighting a critical role of inferior frontal and parietal cortices in cognitive control.

Although $\mathrm{A} \beta$-related and $\mathrm{AD}$-related changes in cognition have been often focused on EM, it is well documented that impairment of cognitive control is one of early cognitive deficits observed in mild AD patients. Accumulating evidence further suggests that cognitive control impairment can be an early behavioral marker discriminating healthy aging from very mild $\mathrm{AD}$ dementia and predicting later conversion (Balota et al., 2010). Even in cognitively normal elderly, well designed cognitive control measures have been associated with AD biomarkers, such as brain amyloid deposition measured by Pittsburgh Compound B PET and CSF measures, including tau, phosphorylated tau ( $\mathrm{p}$ tau), and $\mathrm{A} \beta 42$, and $\mathrm{AD}$ risk factors, such as apolipoprotein E4 (APOE4) genotypes (Aschenbrenner et al., 2015). These findings from both clinical and cognitive/experimental studies, therefore, clearly indicate that cognitive control impairment occurs early in the progression of $\mathrm{AD}$ and may be indicative of a presymptomatic stage of $\mathrm{AD}$. Our results provide evidence at the neural level supporting these previous behavioral findings.

The significance of detecting early changes in cognitive control and underlying neural correlates may depend on its relevance to other neurocognitive systems that may become more critical to the disease progression with advanced $\mathrm{AD}$ pathology. The cognitive psychology and neuroscience literature has consistently shown that cognitive control is a critical and even prerequisite cognitive component for declarative memory (Jacoby, 1991). Consistent with this view, previous work of cognitive neuroscience of memory using the whole-brain analysis with fMRI commonly showed brain activation beyond the MTLs during EM encoding and retrieval tasks. Not surprisingly, studies assessing functional changes in relation to an $\mathrm{AD}$ risk factor, such as APOE4 genotypes, found brain activation changes in cognitive control regions defined during $n$-back working memory tasks. Wishart et al. (2006) showed that APOE4 carriers demonstrated greater activity during verbal $n$-back working memory tasks. Chen et al. (2013) further found that APOE4 carriers showed a greater increase in activation than $A P O E 4$ noncarriers during low working memory-load tasks compared with baseline activity, while less increases in activation than APOE4 noncarriers during higher working memory-load tasks compared with lower working memory-load tasks. Our findings of $\mathrm{A} \beta$-related activation decreases in higher cognitive control load, therefore, are in line with these previous reports while providing direct evidence of the impact of brain $\mathrm{A} \beta$ deposition on brain activation in frontoparietal cognitive control regions. Future studies linking A $\beta$-related abnormal activity in frontoparietal cognitive control regions and EM deficits will be warranted to elucidate this hypothesized mechanism during the progression of AD pathology.

$\mathrm{A} \beta$-related functional alterations in cognitively normal elderly have been often found in a form of hyperactivation given a similar level of cognitive performance in EM (Sperling et al., 2009; Mormino et al., 2012; Oh and Jagust, 2013; Huijbers et al., 2014). This $\mathrm{A} \beta$-related hyperactivation in cognitively normal elderly is consistent with findings showing hippocampal hyperactivity in amnesic MCI patients (Dickerson et al., 2005; Celone et al., 2006; Bakker et al., 2012) and young presymptomatic individuals who carry the presenilin-1 genetic mutation (Quiroz et al., 2010) and the APOE $\varepsilon 4$ allele (Wishart et al., 2006; Filippini et al., 2009; Chen et al., 2013). A recent study by Gordon et al. (2015), however, examined task-evoked fMRI activations using semantic judgment (i.e., animacy) and Stroop tasks in association with several AD biomarkers in cognitively normal elderly and found a significant association of task-related activation with CSF tau and p-tau for both types of tasks, but not with CSF A $\beta 42 /$ amyloid PET measures. Although behavioral ceiling effects and blocklevel modulations (e.g., incongruent and congruent trials combined) in this study may, in part, explain some inconsistencies between results of Gordon et al.'s study and the present study, it is also possible that other AD biomarkers, such as tau pathology, may affect cognitive control brain regions. Furthermore, it is well recognized that brain regions collectively known as the default mode network (DMN) undergo $\mathrm{A} \beta$-related neural dysfunction as manifested by reduced functional synchrony across the network (Hedden et al., 2009) as well as aberrant increases in deactivation (Sperling et al., 2009). Together, our results of $\mathrm{A} \beta$ related hyperactivation during the single-task and dual-task conditions compared with baseline activity, which topographically overlap with the DMN, corroborate previous findings, while providing novel data showing $\mathrm{A} \beta$-related hyperactivation reaches a breakdown with higher cognitive demands and begins to decrease in the cognitive control regions.

Independent of $A \beta$ deposition, we found age-related increases in frontoparietal activation during task switching. These results are highly consistent with previous findings showing age-related hyperactivation in frontoparietal cortices during working memory tasks compared with young adults with an equivalent level of behavioral performance (Cappell et al., 2010; Oh et al., 2015). Age-related hyperactivation within the common brain regions 
involved in task switching supports the view that age is influencing the efficiency of this network, as proposed by our previous work on neural reserve (Stern, 2009) and as suggested by the compensation-related utilization of neural circuits hypothesis (Reuter-Lorenz and Cappell, 2008). Thus all groups can perform comparably on the task, but the network has to be activated to a greater degree in aging because network efficiency is reduced. This age-related reduction in network efficiency and resulting hyperactivity may continue and even increase with $\mathrm{A} \beta$ deposition until it reaches a breakdown when higher cognitive demand can no longer be accommodated by the neural system critical for the process.

Several limitations of the current study need to be addressed. First, while block-based analyses of the fMRI task allowed us to identify brain regions robustly activated in response to cognitive control demand, we could not pinpoint subprocesses supporting task switching with a finer grain. Considering that cognitive control, as demonstrated in task switching, is thought to be achieved by a combination of multiple cognitive processes, including representation of abstract rules for task context and response-stimulus association, decision making, and action selection, future studies will need to address whether and how subcomponents of cognitive control are more susceptible to age and $\mathrm{A} \beta$ deposition. Second, our data were analyzed cross-sectionally, thereby limiting our ability to predict longitudinal outcomes associated with $\mathrm{A} \beta$-related decreases in right prefrontal activation. Although studies collectively suggest that $A \beta$ deposition present in cognitively normal elderly indicates an increased risk for developing $\mathrm{AD}$, a longitudinal assessment of the relationship between fMRI activation and cognitive trajectories will be warranted to fully evaluate the utility of task-related brain activation measures as an early biomarker of AD. Last, studies have consistently shown that the brain activation and cognition relationship is moderated by individual difference factors, such as life experience, education, and exercise, collectively conceptualized as cognitive reserve (Stern, 2009). Because of the relatively small sample size $(\mathrm{A} \beta+$ older subjects in particular), the current study could not directly examine the effect of these modifying factors on age-related and $\mathrm{A} \beta$-related brain activation changes during task switching. Future studies with a larger sample size will be warranted to address neural consequences of reserve on brain activation during task switching, which will allow us to finely tune in to age-related and $\mathrm{A} \beta$-related changes in task-switching activation accounting for reserve-related activation differences.

In summary, we report a novel finding that with $\mathrm{A} \beta$ deposition, older adults show greater activation in the frontoparietal control regions recruited during cognitive control. However, activity in the right prefrontal cortex, which is critical for cognitive control, decreases with increased cognitive control demand in cognitively normal elderly with $A \beta$ deposition. Independent of $\mathrm{A} \beta$ deposition, age is associated with greater frontoparietal activation during task switching. Dynamic $\mathrm{A} \beta$-related changes in frontoparietal activation with different cognitive control demand may indicate crucial early functional mechanisms during the progression of $\mathrm{A} \beta$ pathology.

\section{References}

Adamczuk K, De Weer AS, Nelissen N, Dupont P, Sunaert S, Bettens K, Sleegers K, Van Broeckhoven C, Van Laere K, Vandenberghe R (2016) Functional changes in the language network in response to increased amyloid beta deposition in cognitively intact older adults. Cereb Cortex 26:358-373. CrossRef Medline

Aschenbrenner AJ, Balota DA, Tse CS, Fagan AM, Holtzman DM, Benzinger TL, Morris JC (2015) Alzheimer disease biomarkers, attentional control, and semantic memory retrieval: synergistic and mediational effects of biomarkers on a sensitive cognitive measure in non-demented older adults. Neuropsychology 29:368-381. CrossRef Medline

Badre D, D'Esposito M (2007) Functional magnetic resonance imaging evidence for a hierarchical organization of the prefrontal cortex. J Cogn Neurosci 19:2082-2099. CrossRef Medline

Bakker A, Krauss GL, Albert MS, Speck CL, Jones LR, Stark CE, Yassa MA, Bassett SS, Shelton AL, Gallagher M (2012) Reduction of hippocampal hyperactivity improves cognition in amnestic mild cognitive impairment. Neuron 74:467-474. CrossRef Medline

Balota DA, Tse CS, Hutchison KA, Spieler DH, Duchek JM, Morris JC (2010) Predicting conversion to dementia of the Alzheimer's type in a healthy control sample: the power of errors in Stroop color naming. Psychol Aging 25:208-218. CrossRef Medline

Becker JA, Hedden T, Carmasin J, Maye J, Rentz DM, Putcha D, Fischl B, Greve DN, Marshall GA, Salloway S, Marks D, Buckner RL, Sperling RA, Johnson KA (2011) Amyloid-beta associated cortical thinning in clinically normal elderly. Ann Neurol 69:1032-1042. CrossRef Medline

Bennett DA, Schneider JA, Arvanitakis Z, Kelly JF, Aggarwal NT, Shah RC, Wilson RS (2006) Neuropathology of older persons without cognitive impairment from two community-based studies. Neurology 66:18371844. CrossRef Medline

Braak H, Braak E (1991) Demonstration of amyloid deposits and neurofibrillary changes in whole brain sections. Brain Pathol 1:213-216. CrossRef Medline

Brass M, von Cramon DY (2004) Selection for cognitive control: a functional magnetic resonance imaging study on the selection of task-relevant information. J Neurosci 24:8847-8852. CrossRef Medline

Braver TS, Reynolds JR, Donaldson DI (2003) Neural mechanisms of transient and sustained cognitive control during task switching. Neuron 39: 713-726. CrossRef Medline

Brett M, Anton J-L, Valabregue R, Poline J-B (2002) Region of interest analysis using an SPM toolbox. Neuroimage 16:1140-1141.

Buschke H, Fuld PA (1974) Evaluating storage, retention, and retrieval in disordered memory and learning. Neurology 24:1019-1025. CrossRef Medline

Cappell KA, Gmeindl L, Reuter-Lorenz PA (2010) Age differences in prefontal recruitment during verbal working memory maintenance depend on memory load. Cortex 46:462-473. CrossRef Medline

Celone KA, Calhoun VD, Dickerson BC, Atri A, Chua EF, Miller SL, DePeau K, Rentz DM, Selkoe DJ, Blacker D, Albert MS, Sperling RA (2006) Alterations in memory networks in mild cognitive impairment and Alzheimer's disease: an independent component analysis. J Neurosci 26: 10222-10231. CrossRef Medline

Chen CJ, Chen CC, Wu D, Chi NF, Chen PC, Liao YP, Chiu HW, Hu CJ (2013) Effects of the apolipoprotein E epsilon4 allele on functional MRI during $\mathrm{n}$-back working memory tasks in healthy middle-aged adults. AJNR Am J Neuroradiol 34:1197-1202. CrossRef Medline

Cole MW, Reynolds JR, Power JD, Repovs G, Anticevic A, Braver TS (2013) Multi-task connectivity reveals flexible hubs for adaptive task control. Nat Neurosci 16:1348-1355. CrossRef Medline

Dale AM, Fischl B, Sereno MI (1999) Cortical surface-based analysis. I. Segmentation and surface reconstruction. Neuroimage 9:179-194. CrossRef Medline

Dickerson BC, Salat DH, Greve DN, Chua EF, Rand-Giovannetti E, Rentz DM, Bertram L, Mullin K, Tanzi RE, Blacker D, Albert MS, Sperling RA (2005) Increased hippocampal activation in mild cognitive impairment compared to normal aging and AD. Neurology 65:404-411. CrossRef Medline

Elman JA, Oh H, Madison CM, Baker SL, Vogel JW, Marks SM, Crowley S, O'Neil JP, Jagust WJ (2014) Neural compensation in older people with brain amyloid-beta deposition. Nat Neurosci 17:1316-1318. CrossRef Medline

Filippini N, MacIntosh BJ, Hough MG, Goodwin GM, Frisoni GB, Smith SM, Matthews PM, Beckmann CF, Mackay CE (2009) Distinct patterns of brain activity in young carriers of the APOE-epsilon4 allele. Proc Natl Acad Sci U S A 106:7209-7214. CrossRef Medline

Fischl B, Salat DH, Busa E, Albert M, Dieterich M, Haselgrove C, van der Kouwe A, Killiany R, Kennedy D, Klaveness S, Montillo A, Makris N, Rosen B, Dale AM (2002) Whole brain segmentation: automated labeling of neuroanatomical structures in the human brain. Neuron 33:341355. CrossRef Medline

Golden CJ (1978) Stroop Color and Word Test. Chicago: Stoelting. 
Gordon BA, Zacks JM, Blazey T, Benzinger TL, Morris JC, Fagan AM, Holtzman DM, Balota DA (2015) Task-evoked fMRI changes in attention networks are associated with preclinical Alzheimer's disease biomarkers. Neurobiol Aging 36:1771-1779. CrossRef Medline

Grober E, Sliwinski M (1991) Development and validation of a model for estimating premorbid verbal intelligence in the elderly. J Clin Exp Neuropsychol 13:933-949. CrossRef Medline

Hardy J, Selkoe DJ (2002) The amyloid hypothesis of Alzheimer's disease: progress and problems on the road to therapeutics. Science 297:353-356. CrossRef Medline

Hedden T, Van Dijk KR, Becker JA, Mehta A, Sperling RA, Johnson KA, Buckner RL (2009) Disruption of functional connectivity in clinically normal older adults harboring amyloid burden. J Neurosci 29:1268612694. CrossRef Medline

Huijbers W, Mormino EC, Wigman SE, Ward AM, Vannini P, McLaren DG, Becker JA, Schultz AP, Hedden T, Johnson KA, Sperling RA (2014) Amyloid deposition is linked to aberrant entorhinal activity among cognitively normal older adults. J Neurosci 34:5200-5210. CrossRef Medline

Jacoby LL (1991) A process dissociation framework: separating automatic from intentional uses of memory. J Mem Lang 30:513-541.

Jagust WJ, Mormino EC (2011) Lifespan brain activity, beta-amyloid, and Alzheimer's disease. Trends Cogn Sci 15:520-526. CrossRef Medline

Johnson SC, Christian BT, Okonkwo OC, Oh JM, Harding S, Xu G, Hillmer AT, Wooten DW, Murali D, Barnhart TE, Hall LT, Racine AM, Klunk WE, Mathis CA, Bendlin BB, Gallagher CL, Carlsson CM, Rowley HA, Hermann BP, Dowling NM, et al. (2014) Amyloid burden and neural function in people at risk for Alzheimer's Disease. Neurobiol Aging 35: 576-584. CrossRef Medline

Johnstone T, Ores Walsh KS, Greischar LL, Alexander AL, Fox AS, Davidson RJ, Oakes TR (2006) Motion correction and the use of motion covariates in multiple-subject fMRI analysis. Hum Brain Mapp 27:779-788. CrossRef Medline

Koechlin E, Ody C, Kouneiher F (2003) The architecture of cognitive control in the human prefrontal cortex. Science 302:1181-1185. CrossRef Medline

Landau SM, Breault C, Joshi AD, Pontecorvo M, Mathis CA, Jagust WJ, Mintun MA (2013) Amyloid-beta imaging with Pittsburgh compound $\mathrm{B}$ and florbetapir: comparing radiotracers and quantification methods. J Nucl Med 54:70-77. CrossRef Medline

Landau SM, Fero A, Baker SL, Koeppe R, Mintun M, Chen K, Reiman EM, Jagust WJ (2015) Measurement of longitudinal beta-amyloid change with $18 \mathrm{~F}$-florbetapir PET and standardized uptake value ratios. J Nucl Med 56:567-574. CrossRef Medline

Lowe VJ, Weigand SD, Senjem ML, Vemuri P, Jordan L, Kantarci K, Boeve B, Jack CR Jr, Knopman D, Petersen RC (2014) Association of hypometabolism and amyloid levels in aging, normal subjects. Neurology 82:19591967. CrossRef Medline

Mattis S (1988) Dementia Rating Scale (DRS). Odessa, FL: Psychological Assessment Resources.

Miller EK, Cohen JD (2001) An integrative theory of prefrontal cortex function. Annu Rev Neurosci 24:167-202. CrossRef Medline

Mintun MA, Larossa GN, Sheline YI, Dence CS, Lee SY, Mach RH, Klunk WE, Mathis CA, DeKosky ST, Morris JC (2006) [11C]PIB in a nondemented population: potential antecedent marker of Alzheimer disease. Neurology 67:446-452. CrossRef Medline

Mormino EC (2014) The relevance of beta-amyloid on markers of Alzhei- mer's disease in clinically normal individuals and factors that influence these associations. Neuropsychol Rev 24:300-312. CrossRef Medline

Mormino EC, Smiljic A, Hayenga AO, Onami SH, Greicius MD, Rabinovici GD, Janabi M, Baker SL, Yen IV, Madison CM, Miller BL, Jagust WJ (2011) Relationships between beta-amyloid and functional connectivity in different components of the default mode network in aging. Cereb Cortex 21:2399-2407. CrossRef Medline

Mormino EC, Brandel MG, Madison CM, Marks S, Baker SL, Jagust WJ (2012) $\mathrm{A} \beta$ deposition in aging is associated with increases in brain activation during successful memory encoding. Cereb Cortex 22:1813-1823. CrossRef Medline

Nelissen N, Vandenbulcke M, Fannes K, Verbruggen A, Peeters R, Dupont P, Van Laere K, Bormans G, Vandenberghe R (2007) Abeta amyloid deposition in the language system and how the brain responds. Brain 130: 2055-2069. CrossRef Medline

Oh H, Jagust WJ (2013) Frontotemporal network connectivity during memory encoding is increased with aging and disrupted by beta-amyloid. J Neurosci 33:18425-18437. CrossRef Medline

Oh H, Habeck C, Madison C, Jagust W (2014) Covarying alterations in Abeta deposition, glucose metabolism, and gray matter volume in cognitively normal elderly. Hum Brain Mapp 35:297-308. CrossRef Medline

Oh H, Steffener J, Razlighi QR, Habeck C, Liu D, Gazes Y, Janicki S, Stern Y (2015) Abeta-related hyperactivation in frontoparietal control regions in cognitively normal elderly. Neurobiol Aging 36:3247-3254. CrossRef Medline

Power JD, Cohen AL, Nelson SM, Wig GS, Barnes KA, Church JA, Vogel AC, Laumann TO, Miezin FM, Schlaggar BL, Petersen SE (2011) Functional network organization of the human brain. Neuron 72:665-678. CrossRef Medline

Quiroz YT, Budson AE, Celone K, Ruiz A, Newmark R, Castrillón G, Lopera F, Stern CE (2010) Hippocampal hyperactivation in presymptomatic familial Alzheimer's disease. Ann Neurol 68:865-875. CrossRef Medline

Reitan R (1978) Manual for administration of neuropsychological test batteries for adults and children. Tucson, AZ: Reitan Neuropsychology Laboratories.

Reuter-Lorenz PA, Cappell K (2008) Neurocognitive aging and the compensation hypothesis. Curr Dir Psychol Sci 18:177-182.

Selkoe DJ (2003) Folding proteins in fatal ways. Nature 426:900-904. CrossRef Medline

Sperling RA, Laviolette PS, O'Keefe K, O’Brien J, Rentz DM, Pihlajamaki M, Marshall G, Hyman BT, Selkoe DJ, Hedden T, Buckner RL, Becker JA, Johnson KA (2009) Amyloid deposition is associated with impaired default network function in older persons without dementia. Neuron 63: 178-188. CrossRef Medline

Stern Y (2009) Cognitive reserve. Neuropsychologia 47:2015-2028. CrossRef Medline

Waskom ML, Kumaran D, Gordon AM, Rissman J, Wagner AD (2014) Frontoparietal representations of task context support the flexible control of goal-directed cognition. J Neurosci 34:10743-10755. CrossRef Medline

Wechsler D (1997) Wechsler Adult Intelligence Scale-III. San Antonio, TX: Psychological.

Wishart HA, Saykin AJ, Rabin LA, Santulli RB, Flashman LA, Guerin SJ, Mamourian AC, Belloni DR, Rhodes CH, McAllister TW (2006) Increased brain activation during working memory in cognitively intact adults with the APOE epsilon4 allele. Am J Psychiatry 163:1603-1610. CrossRef Medline 